ARTICLE

\title{
Lung volume reduction in chronic obstructive pulmonary disease
}

\author{
M J Vorster, MB ChB, MRCP (UK), MMed (Int), FCP (SA); \\ C F N Koegelenberg, MB ChB, MMed (Int), FCP (SA), FRCP (UK), Cert Pulmonology (SA), PhD
}

Division of Pulmonology, Department of Medicine, Faculty of Medicine and Health Sciences, Stellenbosch University and Tygerberg Academic Hospital, Cape Town, South Africa

Corresponding author: C F N Koegelenberg (coeniefn@sun.ac.za)

\begin{abstract}
Pathognomonic features of advanced emphysema include a markedly reduced alveolar surface area due to the formation of blebs and bullae and significantly reduced elastic recoil. The aim of lung volume reduction, which can be achieved by either surgery or endoscopic techniques, is volume loss of the targeted, diseased region(s) and redirecting airflow to less affected regions.

Lung volume reduction surgery (LVRS) entails reducing the lung volume by wedge excision of emphysematous tissue. LVRS carries significant morbidity and mortality, but can offer survival benefit and increased exercise capacity in selected patients with predominantly upper-lobe emphysema and low exercise capacity.

Endoscopic lung volume reduction (ELVR) refers to bronchoscopically inducing volume loss to improve pulmonary mechanics and compliance, thereby reducing the work of breathing. Globally, this technique is increasingly used as treatment for advanced emphysema with the objective of obtaining similar functional advantages to surgical lung volume reduction, while decreasing risks and costs. Current evidence suggests that patients with either homogeneous or heterogeneous disease may benefit from ELVR.

It remains paramount that a systematic approach is followed and selection criteria are met, given the high costs and potential complications related to both LVRS and ELVR.
\end{abstract}

S Afr Med J 2015;105(9):791. DOI:10.7196/SAMJnew.8427

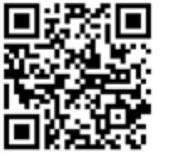

Pathognomonic features of advanced emphysema include a markedly reduced alveolar surface area due to the formation of blebs and bullae and significantly reduced elastic recoil of the lung. ${ }^{[1,2]}$ Early airway closure occurs during expiration, with resultant air trapping and hyperinflation. Consequently, the range of expansion of preserved areas of lung tissue decreases. Furthermore, air trapping and hyperinflation place the diaphragm at a mechanical disadvantage owing to its flattened configuration. These processes in combination lead to refractory dyspnoea. ${ }^{[1]}$

The aim of lung volume reduction (whether done surgically or endoscopically) is to achieve volume loss of the targeted, diseased region(s) and to redirect airflow to less affected areas. ${ }^{[2]}$ This decreases dynamic hyperinflation, and improves diaphragmatic and chest wall mechanics. In theory, the remaining lung tissue has better elastic properties that can restore the outward radial pull on the small airways, thereby reducing airflow limitation. Reducing inhomogeneity of regional ventilation and perfusion may lead to improved ventilation/perfusion matching.

Lung volume reduction can in essence be achieved by either surgical or endoscopic techniques. This article aims to provide the practising general practitioner with an overview of the practical aspects of and current evidence for the use of the various techniques in South Africa (SA). The general indications and contraindications for lung volume reduction are summarised in Table 1.

\section{Lung volume reduction surgery}

Lung volume reduction surgery (LVRS) entails reducing the lung volume by wedge excision of emphysematous tissue. It is most often performed as a bilateral procedure, but occasionally as a unilateral one. Examples of the latter would include cases of severely asymmetrical emphysema, contralateral pleurodesis, contralateral thoracotomy and haemodynamic instability or massive air leak during the first side of a planned bilateral procedure.

The National Emphysema Treatment Trial (NETT) is still the largest randomised trial of LVRS. It compared the benefits of LVRS with maximal medical therapy in $>1000$ patients with advanced emphysema. ${ }^{[3]}$ Within the first few months of starting the trial, a high risk of death ( $16 \%$ v. $0 \%$ controls) was identified in a subgroup of patients with a forced expiratory volume in 1 second $\left(\mathrm{FEV}_{1}\right)<20 \%$ of predicted and either homogeneous emphysema or a diffusing capacity for carbon monoxide $\left(\mathrm{DL}_{\mathrm{CO}}\right)<20 \%$ of predicted. Patients with these characteristics were subsequently excluded from enrolment in NETT. Among patients without these high-risk characteristics, the 30-day mortality rate was $2.2 \%$ in the LVRS group, compared with $0.2 \%$ in the control group $(p<0.001)$. At 2 years, total mortality among non-high-risk patients did not differ between the LVRS and medical therapy groups. The effect of LVRS on exercise capacity was modest, and the investigators concluded that LVRS does not confer a survival advantage over medical therapy. Only in a subgroup of patients with predominantly upper-lobe emphysema and low exercise capacity, was LVRS shown to reduce long-term mortality.

There has been a significant decline in the number of LVRSs performed, both locally and internationally, mostly because of the modest benefit, strict selection criteria, morbidity and mortality associated with major thoracic surgery in patients with compromised pulmonary and cardiovascular reserves and the advent of endoscopic lung volume reduction (ELVR). ${ }^{[2]}$

\section{Endoscopic lung volume reduction Rationale}

The use of ELVR as a minimally invasive procedure with significantly lower morbidity and mortality than surgery is fast becoming a 
Table 1. General indications and contraindications for lung volume reduction in patients with stable emphysema

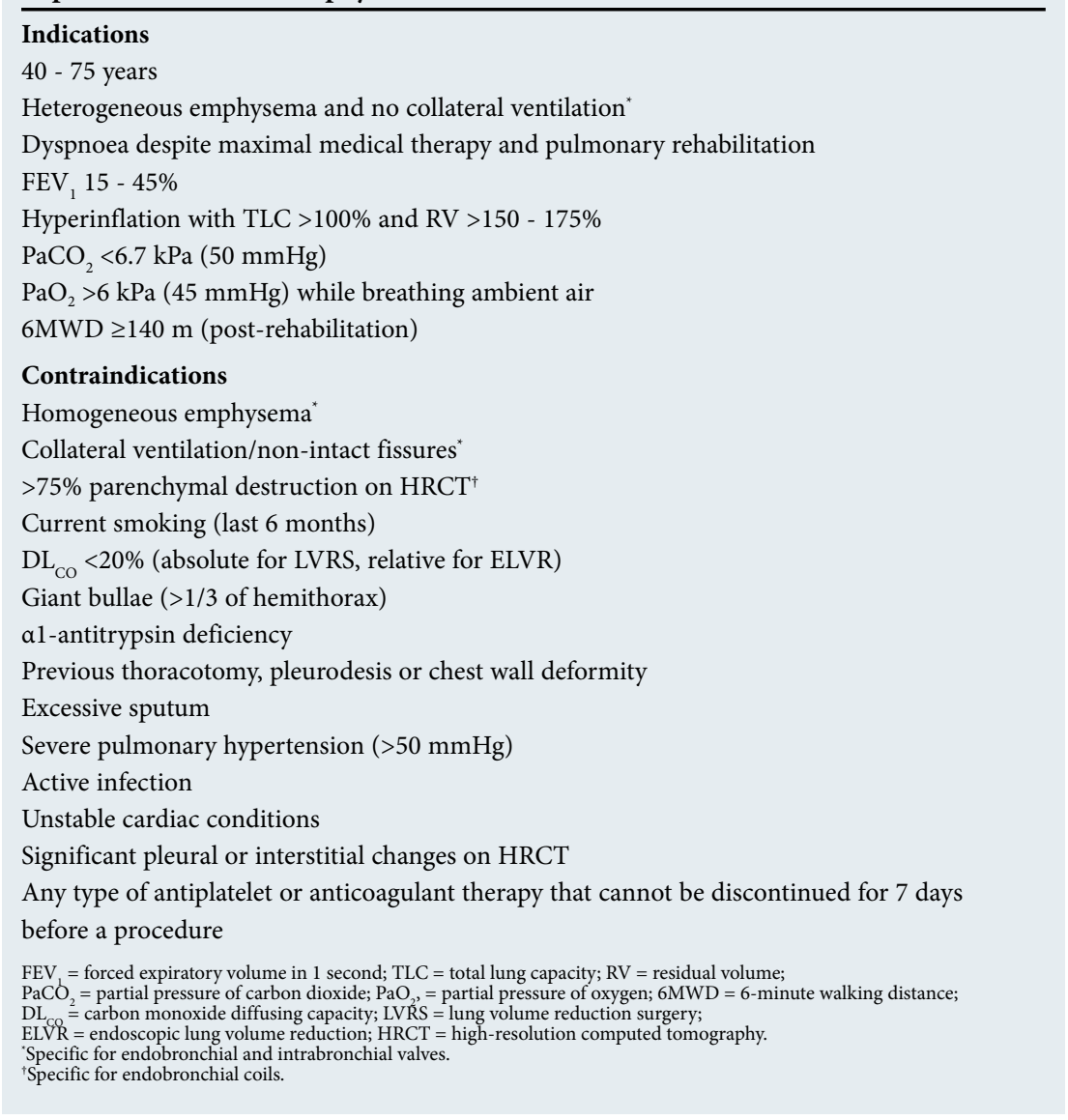

new treatment modality for patients with severe emphysema. The objective is to reduce the risks and costs of surgery and also to achieve comparable physiological benefits. The procedure involves a standard endoscopic technique with endobronchial deployment of the device, which decreases the volume of the distal lung segments, thereby improving pulmonary mechanics and compliance. ${ }^{[2]}$ By reducing airflow limitation and the work of breathing, the result is an almost instantaneous improvement in symptoms - most notably dyspnoea. The two most commonly used devices are endobronchial valves and coils.

\section{Valves}

One-way bronchial valve implantations have been available for use for over a decade, with the greatest clinical experience worldwide. Several clinical trials have been performed, leading to expanded knowledge and expertise with this technology, but the recently completed STELVIO-trial provided the strongest evidence for use of valves in patients without collateral ventilation. ${ }^{[4]}$ Unidirectional valves allow one-way passage of air and secretions from the distal bronchus, thus preventing postobstructive infectious complications. By preventing the entrance of air during inspiration it causes atelectasis of the distal lung segments and a functional lung volume reduction.

The success of the valves depends on whether there is complete collapse of the distal lung segments with the most severe disease, which is determined by two characteristics of emphysema, i.e. the degree of homogeneity and the presence of collateral ventilation. The valves are only effective if there is inhomogeneous emphysema as assessed on chest computed tomography (CT) scanning, either by visual inspection of the parenchyma or using specifically designed automated quantification software. ${ }^{[2]}$ Furthermore, valves fail to induce collapse when the affected portion of the lung has collateral ventilation. This is a normal physiological phenomenon in many individuals, but significant interlobar collateral ventilation subverts the deflating effect of endobronchial blocking devices. Therefore, before placement of a valve, both homogeneous emphysema and collateral ventilation need to be excluded. Moreover, unilateral (compared with bilateral ELVR) valve placement was found to have a better outcome. ${ }^{[5-8]}$

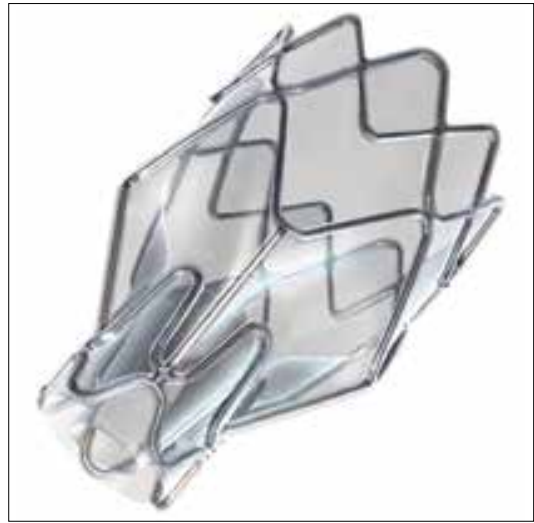

Fig. 1. An endobronchial (Zephyr) valve.

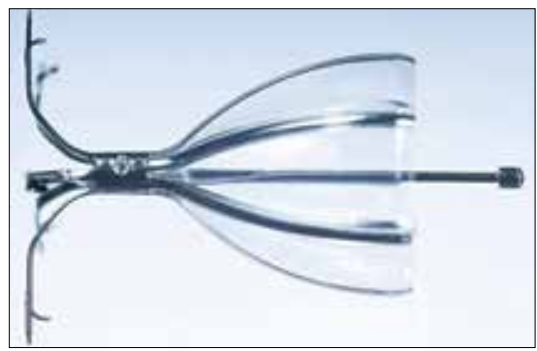

Fig. 2. An intrabronchial (IBV) valve.

There are currently two commercially available devices available in SA: Zephyr endobronchial valves (Pulmonx Inc., USA, Fig. 1) and IBV intrabronchial valves (Olympus Respiratory America, USA, Fig. 2). Both devices are selfexpanding and delivered using a catheter that is introduced through the working channel of a flexible bronchoscope. ${ }^{[2]}$ The most common reported adverse events experienced with endobronchial valve placement have been pneumothoraces (5 - 10\%), mild haemoptysis (2 - 6\%) and exacerbations of underlying chronic obstructive pulmonary disease (COPD) (8 $40 \%) .^{[4-7]}$

\section{Coils}

While valves have been used for more than a decade, coils have only recently been introduced, with the first coils inserted in SA as recently as 2014. Coils (RePneu, BTG Inc., USA) are nitinol devices (Fig. 3) designed to be deployed into a straight airway, and thereafter to resume their preformed shape. This conformational shape change after deployment results in parenchymal retraction with associated volume loss, while maintaining airway patency. ${ }^{[9]}$ The device is currently available in three lengths to accommodate different-sized airways. The coils are implanted via a flexible bronchoscope under general anaesthesia or conscious sedation and fluoroscopic guidance using a proprietary delivery system. Coils, in theory, not only cause lung volume reduction but also reduce airflow limitation by retensioning 


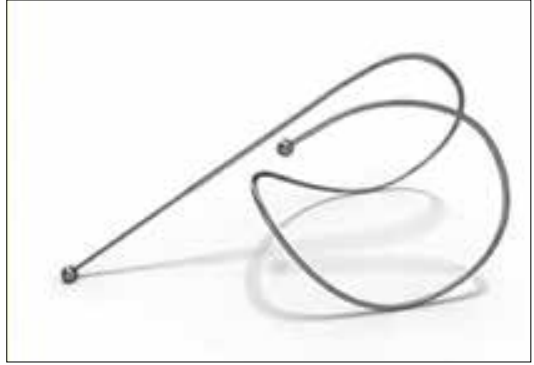

Fig. 3. An endobronchial (RePneu) coil.

the remaining airway network and tethering of the small airways, preventing collapse on expiration.

Current evidence suggests that candidates with both heterogeneous and homogeneous emphysema can experience clinically significant benefit from ELVR using coils. This benefit is obtained regardless of the presence of collateral ventilation or complete lobar collapse after the procedure. However, it requires that $<25 \%$ of the total lung parenchyma be affected by radiological emphysemabeforeinsertion. ${ }^{[10,11]}$ Approximately $75-80 \%$ of patients will experience minimal clinically important differences in lung function and quality of life, while mild haemoptysis of $<5 \mathrm{~mL}(50-75 \%)$, exacerbations of COPD (5 - 12\%), mild chest discomfort (15 - 50\%) and infrequent pneumothoraces (3\%) are described as adverse events. ${ }^{[10,11]}$ A recent report on the 3-year follow-up data of 38 patients who underwent ELVR using coils suggested that the treatment was safe; no late pneumothoraces, coil migrations or unexpected adverse events occurred. ${ }^{[12]}$ Although clinical benefit gradually declined over time, 3 years after treatment approximately $50 \%$ of the patients maintained improvement in 6-minute walking distance, dyspnoea and quality of life scores.

\section{Other devices/procedures}

Synthetic polymeric foam (Aeris Therapeutics Biological, USA) has been used to obtain atelectasis, but a recent study, which was prematurely terminated, raised some safety concerns. ${ }^{[13]}$ This technology is currently not available in SA. Bronchoscopic thermal vapour ablation (BTVA, Uptake Medical Corporation, USA) uses heated water vapour delivered to emphysematous lung parenchyma within a targeted region. The vapour induces an inflammatory reaction with subsequent fibrosis, resulting in lung volume reduction within 8 - 12 weeks. In a multicentre trial in Europe and Australia 44 patients with severe upper-lobe-predominant emphysema were treated by unilateral BTVA in a single procedural setting - an effective treatment with associated clinical benefit independent of collateral ventilation. ${ }^{[14]}$

\section{Future of lung volume reduction in South Africa}

Current evidence suggests that not all classes and phenotypes of emphysema will benefit from ELVR, and that individual techniques may benefit different subgroups of patients. ${ }^{[6,90]}$ Only a few centres in SA currently have the capacity to properly evaluate prospective candidates and potentially offer LVRS and/or ELVR in appropriate cases. The high cost of these interventions makes careful patient selection imperative to prevent wasteful insertion in those unlikely to gain clinical benefit.

The initial screening for suitable candidates should be performed at subspecialist (pulmonologist) level in SA, and on patients with stable disease and no recent exacerbations. Routine special investigations should include high-resolution CT (to estimate heterogeneity, fissure integrity and degree of tissue destruction, and evaluate for possible underlying lung cancer), full pulmonary function testing, arterial blood gas sampling and echocardiography (to exclude pulmonary hypertension) ${ }^{[10]}$ Lung volume reduction should not be offered to active smokers, patients with pulmonary hypertension, unstable cardiac pathology, active respiratory infections, very poor exercise tolerance, without clear evidence of hyperinflation, and those on antiplatelet or anticoagulant therapy that cannot be discontinued for 7 days prior to the procedure. ${ }^{[10,11,15]}$

Appropriate or borderline candidates should be referred to a centre with the capacity to evaluate, treat and follow up, including the managing of complications related to LVRS or ELVR and removal of devices if required.

\section{Conclusion}

A well-structured evidence-based approach to ELVR, including initial screening and subsequent referral to a specialised centre, is important to avoid inappropriate use of devices, which may be both wasteful and harmful. Appropriate candidates with marked hyperinflation and relatively preserved lung parenchyma are more likely to benefit from ELVR with bilateral coils, irrespective of the collateral ventilation and heterogeneity of the disease. By contrast, patients with heterogeneous disease and no collateral ventilation are more likely to benefit from unilateral ELVR with valves, aiming to achieve complete lobar collapse. LVRS should be reserved for patients with heterogeneous disease who have collateral ventilation and an acceptable operative risk profile.

Both LVRS and ELVR are currently available in SA, but there are no head-tohead studies comparing LVRS with ELVR or the various techniques and devices available to perform ELVR, and there are no official guidelines from any of the major thoracic societies. A task group of the Assembly on Interventional Pulmonology of the South African Thoracic Society has extensively reviewed all relevant publications and consulted international experts on the use of ELVR in SA in the form of local recommendations. ${ }^{[16]}$

\section{References}

1. McDonough J, Yuan R, Suzuki M, et al. Small-airway obstruction and emphysema in chronic obstructive pulmonary disease. N Engl J Med 2011;365(17):1567-1575. [http://dx.doi. org/10.1056/NEJMoa1 106955]

2. Gasparini S, Zuccatosta L, Bonifazi M, Bolliger CT. Bronchoscopic treatment of emphysema: State of the art. Respiration 2012;84(3):250-263. [http://dx.doi.org/10.1159/000341171]

3. Fishman A Martinez F Naunheim $K$, et al A randomized trial Fishman A, Martinez F, Naunheim K, et al. A randomized trial comparing lung-volume-reduction surgery with medical therapy for severe emphysema. N Engl J Med 2003;348(21):2059-2073.

4. Klooster K, ten Hacken N, Hartman J, Kerstjens H, van Rikxoort E, Slebos D. Endobronchial valve treatment versus standard medical care in patients with emphysema without interlobar collateral ventilation (The STELVIO-Trial). Am J Respir Crit Care Med 2015;191(9):A6312.

5. Shah P, Slebos D, Cardoso P, et al. Bronchoscopic lung-volume reduction with Exhale airway stents for emphysema (EASE trial): Randomised, sham-controlled, multicentre trial. Lancet 2011;378(9795):997-1005. [http://dx.doi.org/10.1016/S01406736(11)61050-7]

6. Sciurba FC, Ernst A, Herth FIF, et al. A randomized study of endobronchial valves for advanced emphysema $\mathrm{N}$ Engl J ed 2010:363(13):1233-1244. [http:/d hysema. N Engl J Med 2010;363(13):1233-1244. [http://dx.doi.org/10.1056/
NEJMoa0900928] NEJMoa0900928

Mor Z, Leventhal A, Diacon AH, Finger R, Schoch OD. Tuberculosis screening in immigrants from high-prevalence countries: Interview first or chest radiograph first? A pro/con debate. Respirology 2013;18(3):432-438. [http://dx.doi.org/10.1111/resp.12054]

8. Herth FJF, Noppen M, Valipour A, et al. Efficacy predictors of lung volume reduction with Zephyr valves in a European cohort. Eur Respir J 2012;39(6):1334-1342. [http://dx.doi. org/10.1183/09031936.00161611]

9. Herth FJF, Eberhard R, Gompelmann D, Slebos D-J, Ernst A. Bronchoscopic lung volume reduction with a dedicated coil: A clinical pilot study. Ther Adv Respir Dis 2010;4:225-231. [http:// dx.doi.org/10.1177/1753465810368553]

10. Deslee G, Klooster K, Hetzel M, et al. Lung volume reduction coil treatment for patients with severe emphysem. A Europen coil treatment for patients with severe emphysema: A European multicentre trial. Thorax 2014;69(11):980-986. [http://dx.doi.
org/10.1136/thoraxinl-2014-205221] org/10.1136/thoraxjnl-2014-205221]

Kloster K, ten Hen NHT, Franz I, Kerstjens HM, van Rikxoort EM, Slebos D-J. Lung volume reduction coil treatment in chronic obstructive pulmonary disease patients with homogeneous emphysema: A prospective feasibility trial. Respiration 2014;88(2):116-125. [http://dx.doi.org/10.1159/000362522]

12. Hartman J, Klooster K, Gortzak K, ten Hacken N, Slebos D. Long-term follow-up after bronchoscopic lung volume reduction treatment with coils in patients with severe emphysema. Respirology 2015;20(2):319-326. [http://dx.doi.org/10.1111/resp.12435]

13. Come C, Kramer M, Dransfield M, et al. A randomised trial of lung sealant versus medical therapy for advanced emphysema. Eur Respir J 2015:1-12. [http://dx.doi.org/10.1183/09031936.00205614]

4. Snell G, Herth F, Hopkins P, et al. Bronchoscopic thermal vaell G, Herth F, Hopkins P, et al. Bronchoscopic thermal vapour ablation therapy in the management of heterogeneous emphysema. Eur Respir J 2012;39(6):1326-1333. [http://dx.doi.
org/10.1183/09031936.00092411] org/10.1183/09031936.0002

5. Eberhard R, Gompelmann D, Schuhmann M, et al. Complete unilateral vs partial bilateral endoscopic lung volume reduction in patients with bilateral lung emphysema. Chest 2012;142(4):900-908. [http://dx.doi.org/10.1378/chest.11-2886]

16. Koegelenberg CFN, Theron J, Dheda K, et al. Recommendations for endoscopic lung volume reduction in South Africa: Role in emphysema. S Afr Med J (in press). 